\title{
Process Parameters Optimization of Silica Sand Nanoparticles Production Using Low Speed Ball Milling Method
}

\author{
Zulkhairi Rizlan and Othman Mamat \\ Department of Mechanical Engineering, Universiti Teknologi PETRONAS, Bandar Seri Iskandar, 31750 Tronoh, Perak, Malaysia \\ Correspondence should be addressed to Zulkhairi Rizlan; zulkhairi1987@gmail.com
}

Received 7 October 2013; Accepted 20 January 2014; Published 26 February 2014

Academic Editors: Y. Li and L. Mai

Copyright ( 2014 Z. Rizlan and O. Mamat. This is an open access article distributed under the Creative Commons Attribution License, which permits unrestricted use, distribution, and reproduction in any medium, provided the original work is properly cited.

\begin{abstract}
Experiments are designed using Taguchi method to find the optimum parameters for silica sand nanoparticles production using low speed ball milling. Orthogonal array and signal-to-noise ratio are applied to study performance characteristics of machining parameters which are the ball to powder weight ratio, volume of milling jar, and rotation speed. Results obtained from signal-tonoise ratio analysis showed that ball to powder weight ratio is the most influential parameter.
\end{abstract}

\section{Introduction}

Nanoparticles of silica sand have been researched progressively and produced due to the unique features as a result of size reduction. Silica sand nanoparticles have proved to be a very effective additive to polymers by improving durability, strength, and flexibility. Nanosilica is also used as an additive to improve strength and workability of self-compacting and high performance concretes. Nanosilica particles are widely produced by chemical processes. However, chemical synthesis of nanosilica produces high contamination in the final products. As the demand is increasing for higher nanosilica purity, contamination is expected at the minimum level. Other than chemical synthesis, other processes such as precipitation, vaporization at high temperature, sol-gel process, high speed vertical rotating mill, and planetary ball mill are among the most commonly used methods to produce silica sand nanoparticles. The objective of this research is to design a technique of transforming natural Tronoh silica sand to silica sand nanoparticles by using a combination of low speed ball milling and heating processes. It is expected that the technique will be able to produce high purity silica sand nanoparticles of less than $100 \mathrm{~nm}$ consistently.

Taguchi method provides a simple, efficient, and systematic approach to determine the optimum parameters [1]. Compared to the factorial method, instead of testing all possible combinations of parameters available, Taguchi method provides a more simplified way to set up the combination of experiment parameters. The parameters tested in this paper are ball to powder weight ratio (BPR), volume of milling jar, and milling speed.

\section{Parameters Identification}

There are a lot of parameters used in ball milling process. However, the parameters that have been tested most for optimization are the rotation speed and milling time. This indicates that these two parameters play an important role in determining the effectiveness of the milling. As supported by Simoes, ball to powder weight ratio is recognized as one of the most influential parameters, alongside milling time and rotation speed [2]. Zhang et al. believed that the volume of milling medium is the most influential parameter, followed by the rotation speed [3]. The ball to powder weight ratio used by previous works clearly varies from one another. Although majority of the ratios used were in the range of $10: 1$ to $20: 1$, there are works that have used ratio much higher than that, going up to $100: 1$ [4]. Higher ball to powder weight ratio helps increase the particle size reduction rate. However, when the ratio used is too high, there is a possibility that contamination resulting from the collision of grinding balls and inner wall of milling vial will happen. There is no conclusive decision on the best BPR since some work got 
TABLE 1: Parameters selected and levels for each parameter.

\begin{tabular}{lccc}
\hline Parameter & Level 1 & Level 2 & Level 3 \\
\hline Ball to powder weight ratio (BPR) & $20: 1$ & $15: 1$ & $10: 1$ \\
Volume of milling jar & $5.6 \mathrm{~L}$ & $1.8 \mathrm{~L}$ & $1.0 \mathrm{~L}$ \\
Milling speed & $100 \mathrm{rpm}$ & $95 \mathrm{rpm}$ & $90 \mathrm{rpm}$ \\
\hline
\end{tabular}

TABLE 2: Parameter design orthogonal array selector [8].

\begin{tabular}{lcccccccc}
\hline \multirow{2}{*}{ Number of level } & \multicolumn{8}{c}{ Number of parameters $(P)$} \\
& 2 & $\mathbf{3}$ & 4 & 5 & 6 & 7 & 8 & 9 \\
\hline 2 & 4 & $\mathbf{4}$ & 8 & 8 & 8 & 8 & 12 & 12 \\
$\mathbf{3}$ & $\mathbf{9}$ & $\mathbf{9}^{*}$ & $\mathbf{9}$ & $\mathbf{1 8}$ & $\mathbf{1 8}$ & $\mathbf{1 8}$ & $\mathbf{1 8}$ & $\mathbf{2 7}$ \\
4 & $' 16$ & $' 16$ & $' 16$ & '16 & '32 & '32 & '32 & '32 \\
5 & 25 & $\mathbf{2 5}$ & 25 & 25 & 25 & 50 & 50 & 50 \\
\hline
\end{tabular}

the best result when using BPR of $20: 1$ while some got the best result when using BPR of $10: 1$. Other work stated that the BPR should be at least $15: 1$ to yield the best result [5]. Therefore, the BPR used in this research are $10: 1,15: 1$, and $20: 1$.

For volumes of milling jar, due to availability, there are three sizes available which are 5.6 L, 1.8 L, and 1.0 L. Another parameter that has high influence on milling effectiveness is the milling speed. Almost all of the researches used high rotation or milling speed, which is more than $100 \mathrm{rpm}$. In fact, a milling speed as high as $2000 \mathrm{rpm}$ was used as has been demonstrated by Bilgili et al. [6]. Higher milling speed provides higher impact energy exerted by the grinding balls onto the materials and resulted in better milling effectiveness. For milling speed, since previous works usually used high speed ball milling which is more than $100 \mathrm{rpm}$, the best speed for low speed ball mill is unknown. Since higher speed results in higher collision between grinding balls and powder [7], the three highest speeds of the ball mill will be used which are $100 \mathrm{rpm}, 95 \mathrm{rpm}$, and $90 \mathrm{rpm}$. Table 1 shows the parameters selected and the levels for each parameter.

There are three parameters and levels selected for optimum parameters identification. Taguchi method uses an orthogonal array to design the experiments. From the parameter design orthogonal array selector shown in Table 2, the selected array is level 9 (L9) array. Table 3 shows the level 9 (L9) array parameters configuration while Table 4 shows the actual parameters and levels for each experiment in L9 array.

\section{Experimental Setup}

Ball milling is a method for fine powder grinding and fabrication of submicron or nanostructured powder materials [9]. Ball mill is used for the purpose of grinding all kind of mines and materials $[10,11]$. Throughout the years, it has proved to be an effective and simple technique to produce nanocrystalline powders and the possibility of obtaining large quantity of materials [12]. The grinding jars are arranged eccentrically on sun wheel of planetary ball mill. Grinding balls in grinding jars are subjected to superimposed rotational movements, known as the Coriolis forces. The difference in
TABLE 3: Level 9 array.

\begin{tabular}{lccc}
\hline Experiment & Parameter 1 & Parameter 2 & Parameter 3 \\
\hline 1 & 1 & 1 & 1 \\
2 & 1 & 2 & 2 \\
3 & 1 & 3 & 3 \\
4 & 2 & 1 & 2 \\
5 & 2 & 2 & 3 \\
6 & 2 & 3 & 1 \\
7 & 3 & 1 & 3 \\
8 & 3 & 2 & 1 \\
9 & 3 & 3 & 2 \\
\hline
\end{tabular}

TABLE 4: Actual parameters and levels in L9 array.

\begin{tabular}{lccc}
\hline Experiment & BPR & $\begin{array}{c}\text { Volume of } \\
\text { milling jar }\end{array}$ & Rotation speed \\
\hline 1 & $20: 1$ & $5.6 \mathrm{~L}$ & $100 \mathrm{rpm}$ \\
2 & $20: 1$ & $1.8 \mathrm{~L}$ & $95 \mathrm{rpm}$ \\
3 & $20: 1$ & $1.0 \mathrm{~L}$ & $90 \mathrm{rpm}$ \\
4 & $15: 1$ & $5.6 \mathrm{~L}$ & $95 \mathrm{rpm}$ \\
5 & $15: 1$ & $1.8 \mathrm{~L}$ & $90 \mathrm{rpm}$ \\
6 & $15: 1$ & $1.0 \mathrm{~L}$ & $100 \mathrm{rpm}$ \\
7 & $10: 1$ & $5.6 \mathrm{~L}$ & $90 \mathrm{rpm}$ \\
8 & $10: 1$ & $1.8 \mathrm{~L}$ & $100 \mathrm{rpm}$ \\
9 & $10: 1$ & $1.0 \mathrm{~L}$ & $95 \mathrm{rpm}$ \\
\hline
\end{tabular}

speed between grinding balls and grinding jars produces interaction between frictional and impact forces, releasing high dynamic energies. The interplay between these forces produces high and very effective degree of size reduction of planetary ball mill [13].

The ball mill used is US Stoneware ball mill with maximum speed of $100 \mathrm{rpm}$. Grinding jars used are Roalox Alumina-Fortified Grinding Jars. They are made of wearresistant chemical porcelain fortified with alumina that provides nearly 4 times the wear-life of porcelain jars. The highstrength, impact-resistant body minimizes chipping and breakage, reducing the possibility of powder contamination [14]. The grinding balls used are made from zirconia with diameter of $10 \mathrm{~mm}$.

\section{Experimental Procedures}

Natural silica sand was taken from Tronoh at a location away from developed areas to avoid mixture between natural silica sand and construction sand. The sand was first being washed to remove impurities and dried in oven at $120^{\circ} \mathrm{C}$ for 1 hour. Then, the sand was meshed using Sieve Shaker to a size between $425 \mu \mathrm{m}$ and $600 \mu \mathrm{m}$. The sand was then inserted into the grinding jar together with the grinding balls and milled for 2 hours. After 2 hours of milling, the sand was meshed for the size below $425 \mu \mathrm{m}$. The purpose of meshing step is to remove impurities and hard agglomerates that may build up during milling [15]. After the sand has been meshed, it is again being dried in oven at $120^{\circ} \mathrm{C}$ for 1 hour. 
TABLE 5: Parameters optimization experiments results.

\begin{tabular}{|c|c|c|c|c|c|c|c|}
\hline \multirow{2}{*}{ Experiment } & \multirow{2}{*}{$\mathrm{BPR}$} & \multirow{2}{*}{ Jar volume } & \multirow{2}{*}{ Rotation speed } & \multicolumn{4}{|c|}{ Particle size $(\mu \mathrm{m})$} \\
\hline & & & & Sample 1 & Sample 2 & Sample 3 & Mean \\
\hline 1 & $20: 1$ & $5.6 \mathrm{~L}$ & $100 \mathrm{rpm}$ & 3.017 & 3.002 & 2.979 & 2.999 \\
\hline 2 & $20: 1$ & $1.8 \mathrm{~L}$ & $95 \mathrm{rpm}$ & 2.901 & 2.890 & 2.849 & 2.880 \\
\hline 3 & $20: 1$ & $1.0 \mathrm{~L}$ & $90 \mathrm{rpm}$ & 2.906 & 2.886 & 2.876 & 2.893 \\
\hline 4 & $15: 1$ & $5.6 \mathrm{~L}$ & $95 \mathrm{rpm}$ & 5.304 & 5.213 & 5.215 & 5.244 \\
\hline 5 & $15: 1$ & $1.8 \mathrm{~L}$ & $90 \mathrm{rpm}$ & 4.652 & 4.633 & 4.606 & 4.630 \\
\hline 6 & $15: 1$ & $1.0 \mathrm{~L}$ & $100 \mathrm{rpm}$ & 4.927 & 4.972 & 4.955 & 4.951 \\
\hline 7 & $10: 1$ & $5.6 \mathrm{~L}$ & $90 \mathrm{rpm}$ & 10.105 & 9.976 & 10.040 & 10.040 \\
\hline 8 & $10: 1$ & $1.8 \mathrm{~L}$ & $100 \mathrm{rpm}$ & 7.941 & 8.001 & 7.967 & 7.969 \\
\hline 9 & $10: 1$ & $1.0 \mathrm{~L}$ & $95 \mathrm{rpm}$ & 6.341 & 6.358 & 6.331 & 6.343 \\
\hline
\end{tabular}

The meshing and drying process are repeated every 2 hours of milling until the total milling time has reached 10 hours. The mesh sizes used were $425 \mu \mathrm{m}, 212 \mu \mathrm{m}, 150 \mu \mathrm{m}$, and $63 \mu \mathrm{m}$. After 10 hours of milling, the silica sand was analyzed using Malvern Instruments Mastersizer 2000 particle size analyzer for particle size and size distribution.

\section{Parameters Optimization}

After the experiments have been done, results for Taguchi method experiments are analyzed using particle size analyzer and are shown in Table 5. The evaluation criterion for the milling process is the particle size.

From particle size analyzer results, the value taken for particle size comparison is $d(0.5)$, the median diameter. Median diameter is the size in microns at which $50 \%$ of the sample is smaller and $50 \%$ is larger [16]. It is also known as mass median diameter (MMD). For each experiment, three samples are taken to be analyzed and the average is calculated to get the mean particle size.

\section{Signal-to-Noise Ratio}

To determine the effect of each parameter on the output, the signal-to-noise ratio, or the $\mathrm{SN}$ number, needs to be calculated for each of the experiments conducted. The SN number calculated will determine the optimum parameters and which parameter is the most influential to the result [17]. In the equation below, $y_{i}$ is the mean value which is the result for each experiment and $s_{i}$ is the variance. The signal-to-noise ratio equation derivation is shown from (1) to (3). Consider the following:

$$
\mathrm{SN}_{i}=10 \log \left(\frac{\bar{y}_{i}^{2}}{s_{i}^{2}}\right)
$$

where

$$
\begin{gathered}
\bar{y}_{i}=\frac{1}{N_{i}} \sum_{u=1}^{N_{i}} y_{i, u} \\
s_{i}^{2}=\left(\frac{1}{N_{i}}-1\right) \sum_{u=1}^{N_{i}}\left(y_{i, u}-\bar{y}_{i}\right),
\end{gathered}
$$

TABLE 6: SN ratio for each experiment.

\begin{tabular}{lcccc}
\hline Experiment & BPR & $\begin{array}{c}\text { Volume of } \\
\text { milling jar }\end{array}$ & Milling speed & SN ratio \\
\hline 1 & 1 & 1 & 1 & -9.54 \\
2 & 1 & 2 & 2 & -9.18 \\
3 & 1 & 3 & 3 & -9.21 \\
4 & 2 & 1 & 2 & -14.39 \\
5 & 2 & 2 & 3 & -13.31 \\
6 & 2 & 3 & 1 & -13.89 \\
7 & 3 & 1 & 3 & -20.03 \\
8 & 3 & 2 & 1 & -18.02 \\
9 & 3 & 3 & 2 & -16.04 \\
\hline
\end{tabular}

where $i$ is the experiment number, $u$ is the trial number, and $N_{i}$ is the number of trials for experiment $i$.

For minimizing the performance characteristics, the following equation (3) of SN ratio should be used [18]. SN ratio value calculated for each experiment is shown in Table 6 . Consider the following:

$$
\mathrm{SN}_{i}=-10 \log \sum_{u=1}^{N_{i}} \frac{y_{u}^{2}}{N_{i}} .
$$

After SN ratio for each experiment has been calculated, the average $\mathrm{SN}$ ratio is determined for each parameter and level. The values are then tabulated in Table 7, also known as $\mathrm{SN}$ response table. The range, $R$ (highest $\mathrm{SN}$-lowest $\mathrm{SN}$ ), for each parameter is calculated and compared. The larger the $R$ values for a parameter are, the larger the effect of the parameter on the process is. The same change in signal causes larger effect on the output variable [19].

From Table 7, it is known that the ball to powder weight ratio is the most influential parameter. Volume of milling jar is the second most influential parameter followed by rotation speed. It is also known that, for ball to powder weight ratio parameter, level 1 (BPR 20:1) is the optimum level. For volume of milling jar, the optimum level is level 3 (Volume $1.0 \mathrm{~L}$ ) while for milling speed level $2(95 \mathrm{rpm})$ is the optimum level. Table 8 summarizes the optimum parameters calculated. 
TABLE 7: Signal-to-noise ratio for each parameter and level.

\begin{tabular}{lccc}
\hline Level & $\begin{array}{c}\text { Ball to powder } \\
\text { weight ratio }\end{array}$ & $\begin{array}{c}\text { Volume of } \\
\text { milling jar }\end{array}$ & Rotation speed \\
\hline 1 & $-9.3149^{*}$ & -14.6563 & -13.82 \\
2 & -13.8667 & -13.5097 & $-13.20^{*}$ \\
3 & -18.0367 & $-13.0222^{*}$ & -14.18 \\
Range, $R$ & 8.7218 & 1.6341 & 0.97 \\
Rank & 1 & 2 & 3 \\
\hline
\end{tabular}

* Optimum parameter level.

TABLE 8: Optimum parameters.

\begin{tabular}{lcc}
\hline Parameter & Level & Description \\
\hline Ball to powder weight ratio & 1 & $20: 1$ \\
Volume of milling jar & 3 & $1.0 \mathrm{~L}$ \\
Rotation speed & 2 & $95 \mathrm{rpm}$ \\
\hline
\end{tabular}

\section{Summary}

Taguchi method of experimental design has been applied to optimize multiple process parameters for silica sand nanoparticles production using low speed ball milling method with the L9 array. Results obtained from Taguchi method were analyzed using signal-to-noise ratio for optimum parameters identification. Ball to powder weight ratio is found to be the most influential parameter. The optimum BPR in this paper is $20: 1$, while the optimum volume of milling jar is $1.0 \mathrm{~L}$. It is also known that the optimum rotation speed is $95 \mathrm{rpm}$.

\section{Conflict of Interests}

The authors declare that there is no conflict of interests regarding the publication of this paper.

\section{References}

[1] B. M. Gopalsamy, B. Mondal, and S. Ghosh, "Taguchi method and anova: an approach for process parameters optimization of hard machining while machining hardened steel," Journal of Scientific and Industrial Research, vol. 68, no. 8, pp. 686-695, 2009.

[2] A. D. Rud and A. M. Lakhnik, "Effect of carbon allotropes on the structure and hydrogen sorption during reactive ball-milling of Mg-C powder mixtures," International Journal of Hydrogen Energy, vol. 37, no. 5, pp. 4179-4187, 2012.

[3] F. L. Zhang, M. Zhu, and C. Y. Wang, "Parameters optimization in the planetary ball milling of nanostructured tungsten carbide/cobalt powder," International Journal of Refractory Metals and Hard Materials, vol. 26, no. 4, pp. 329-333, 2008.

[4] G. F. Zhou and H. Bakker, "Atomically disordered nanocrystalline Co2Si by high-energy ball milling," Journal of Physics Condensed Matter, vol. 6, no. 22, pp. 4043-4052, 1994.

[5] O. M. Lemine, M. A. Louly, and A. M. Al-Ahmari, "Planetary milling parameters optimization for the production of $\mathrm{ZnO}$ nanocrystalline," International Journal of Physical Sciences, vol. 5, no. 17, pp. 2721-2729, 2010.
[6] E. Bilgili, R. Hamey, and B. Scarlett, "Production of pigment nanoparticles using a wet stirred mill ith polymeric media," China Particuology, pp. 93-100, 2004.

[7] C. Suryanarayana, "Mechanical alloying and milling," Progress in Materials Science, vol. 46, no. 1-2, pp. 1-184, 2001.

[8] W. Zhao, M. Fang, F. Wu, H. Wu, L. Wang, and G. Chen, "Preparation of graphene by exfoliation of graphite using wet ball milling," Journal of Materials Chemistry, vol. 20, no. 28, pp. 5817-5819, 2010.

[9] A. Mahamat, A. Rani, and P. Husain, "Behavior of Cu-WC-Ti metal composite after using planetary ball milling," International Journal of Engineering and Applied Sciences, pp. 278-282, 2012.

[10] Y. Wang and E. Forssberg, "Production of carbonate and silica nano-particles in stirred bead milling," International Journal of Mineral Processing, vol. 81, no. 1, pp. 1-14, 2006.

[11] M. Hubert, E. Petracovschi, X.-H. Zhang, and L. Calvez, "Synthesis of Germanium-Gallium-Tellurium (Ge-Ga-Te) ceramics by ball-milling and sintering," Journal of the American Ceramic Society, vol. 96, no. 5, pp. 1444-1449, 2013.

[12] P. Álvarez, J. L. S. Llamazares, M. J. Pérez et al., "Microstructural and magnetic characterization of Nd2Fel7 ball milled alloys," Journal of Non-Crystalline Solids, vol. 354, no. 47-51, pp. 5172$5174,2008$.

[13] T. Ahmeda and O. Mamat, "Characterization and properties of copper-silica sand nanoparticle composites," Defect and Diffusion Forum, vol. 319-320, pp. 95-105, 2011.

[14] U. Eşme, "Application of Taguchi method for the optimization of resistance spot welding process," The Arabian Journal For Science and Engineering, vol. 34, no. 2, pp. 519-528, 2009.

[15] S. C. Halim, "Flame spray synthesis of nanoparticles for food applications: synthesis, characterization, potential hazards, cost and scalability," in Proceedings of the AIChE Annual Meeting, Salt Lake City, Utah, USA, 2007.

[16] L. Guoxian, W. Erde, and W. Zhongren, "Effects of ball-milling intensity on the amorphization rate of mixed $\mathrm{Ni}_{50} \mathrm{Ti}_{50}$ powders," Journal of Materials Processing Technology, vol. 51, no. 1-4, pp. 122-130, 1995.

[17] K. Akçay, A. Sirkecioğlu, M. Tatlıerb, O. T. Savaşçı, and A. Erdem-Şenatalar, "Wet ball milling of zeolite HY" Powder Technology, vol. 142, no. 2-3, pp. 121-128, 2004.

[18] M. Hedayati, M. Salehi, R. Bagheri, M. Panjepour, and A. Maghzian, "Ball milling preparation and characterization of poly (ether ether ketone)/surface modified silica nanocomposite," Powder Technology, vol. 207, no. 1-3, pp. 296-303, 2011.

[19] D. Zhang, R. Cai, Y. Zhou, Z. Shao, X.-Z. Liao, and Z.-F. $\mathrm{Ma}$, "Effect of milling method and time on the properties and electrochemical performance of $\mathrm{LiFePO} 4 / \mathrm{C}$ composites prepared by ball milling and thermal treatment," Electrochimica Acta, vol. 55, no. 8, pp. 2653-2661, 2010. 

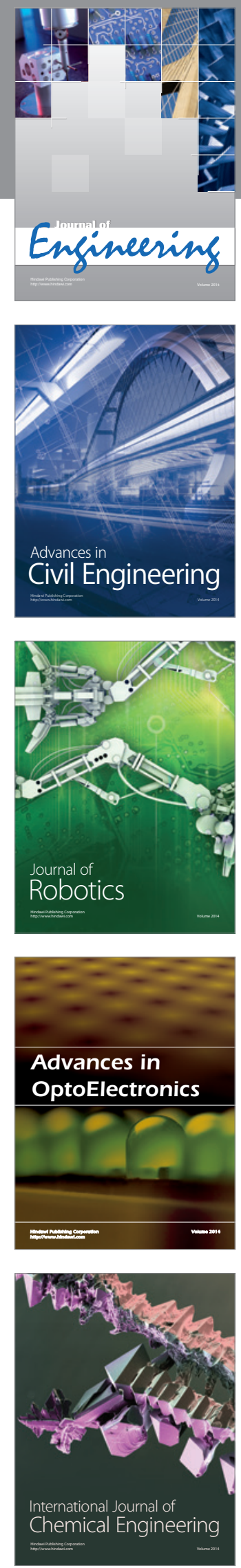

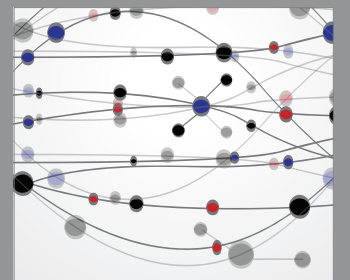

The Scientific World Journal
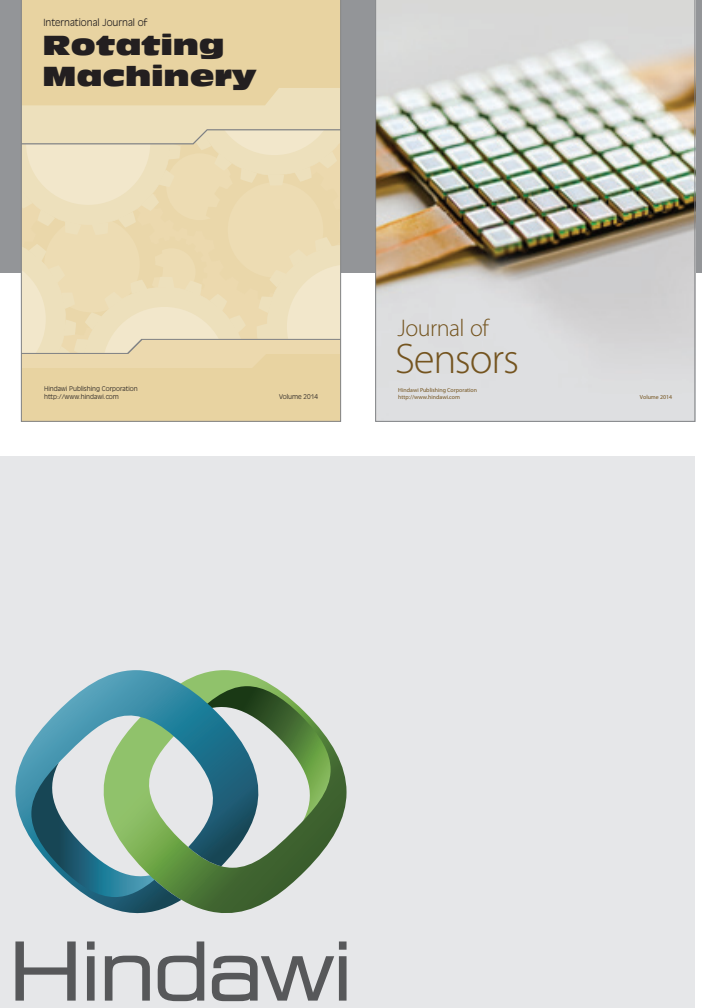

Submit your manuscripts at http://www.hindawi.com
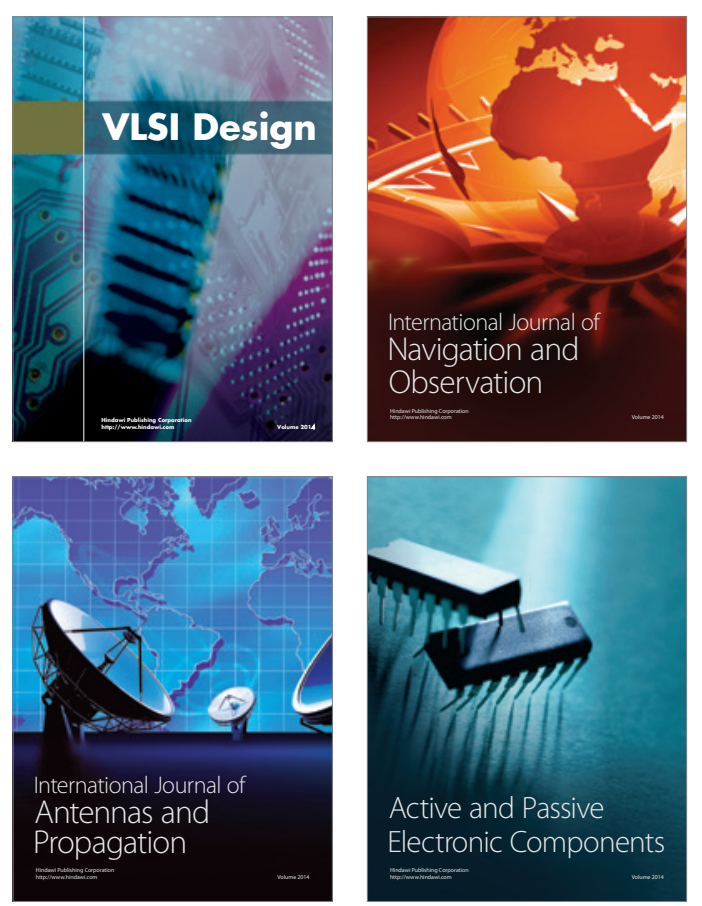
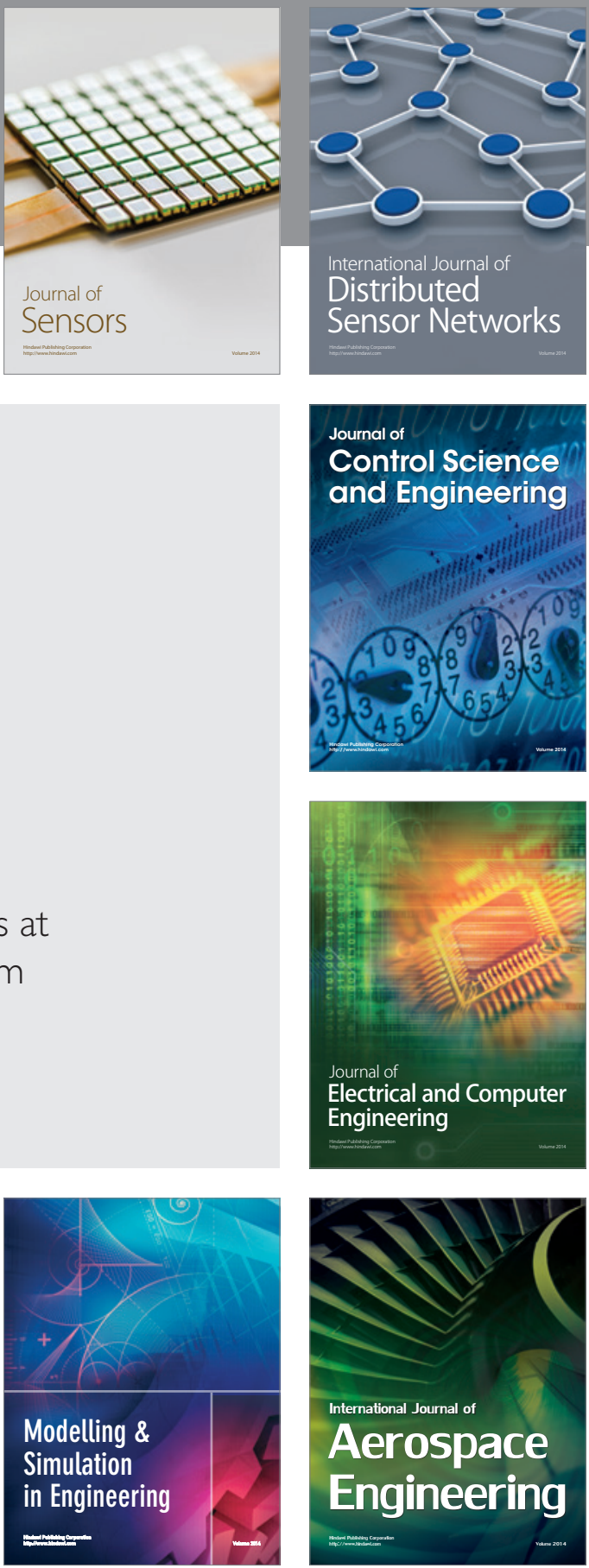

Journal of

Control Science

and Engineering
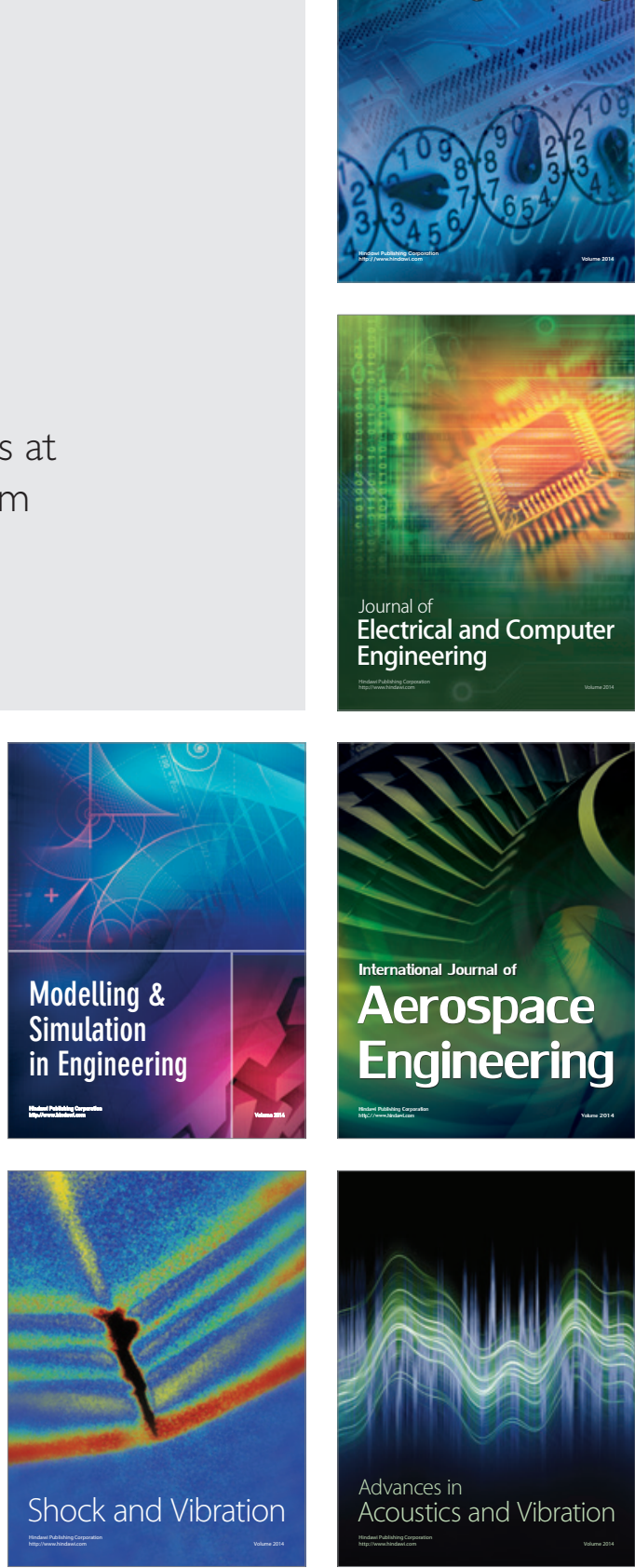